\title{
Adaptation and Normalization Experiments in Speech Recognition for 4 to 8 Year old Children
}

\author{
Daniel Elenius and Mats Blomberg. \\ Department of Speech Music and Hearing \\ KTH, Stockholm, Sweden
}

\begin{abstract}
An experimental offline investigation of the performance of connected digits recognition was performed on children in the age range four to eight years. Poor performance using adult models was improved significantly by adaptation and vocal tract length normalisation but not to the same level as training on children. Age dependent models were tried with limited advantage. A combined adult and child training corpus maintained the performance for the separately trained categories. Linear frequency compression for vocal tract length normalization was attempted but estimation of the warping factor was sensitive to non-speech segments and background noise. Phoneme-based word modeling outperformed the whole word models, even though the vocabulary only consisted of digits.
\end{abstract}

\section{Introduction}

Speech recognition for children is a key to create automatic speech training, language learning, reading tutors, information retrieval, and entertainment applications for children. Children are also a vital resource in the research of adaptation and normalization techniques since their speech differs significantly from that of adults not only in the acoustic domain, but also on the lexical and grammatical levels. However, this paper mainly reports about the performance from an acoustic point of view. For this aim an HMM-based recognizer operating on the small vocabulary of connected digit strings is used.

Observations of formant frequencies of 5-7 year old children being shifted up to $65 \%$ compared to adult formants have been reported [1]. Piece-wise VTLN has improved recognition of child speech when the bandwidth of the signal matches the one used by the recognizer [3]. Linear warping has been combined with spectral shaping in [4]. VTLN was compared to MLLR and constrained MLLR for Italian children [2].

This work extends the age range to younger children than in previous work and compares the performance of VTLN to standard adaptation methods. Baseline results are presented using adult models and models trained on children.

\section{Database}

Adult speech was extracted from the SpeeCon database [5]. The utterances used were read from screen and recorded by a set of microphones sampled at 16 bits and $16 \mathrm{kHz}$. In this investigation only the Sennheiser ME 104 headset was used.

Children were represented by the recordings in the Swedish part of the PF-STAR database [6]. It contains 198 four to eight year old children. Audio prompts were used in favor of text prompts since the youngest children had not yet learnt to read and the older children could not be expected to read fluently. It is likely that their pronunciation was influenced by that of the adult recording leader. This most probably has a normalizing effect on the material and the word error rate for this corpus is expected to be lower than in a practical application. However, the database is judged to be sufficient for comparison between different training, normalization and adaptation procedures which is the focus of this paper.

Recordings were made in a separate room at day-care and after-school centers in the neighborhood of Stockholm. These recordings were made using a Sennheiser ME 104 equipped headset and a MBF Haun, omni-directional microphone. In the current study only the headset signal was used.

The speech signal was sampled at $32 \mathrm{kHz}$ with an amplitude resolution of 24 bits. For most of the experiments in this report, downsampling to $16 \mathrm{kHz}$ and a reduction and 16 bits per sample was performed to harmonize the PF-STAR data and the SpeeCon data. One exception is the experiment with linear frequency VTLN, in which the original $32 \mathrm{kHz}$ sampling rate was used.

\subsection{Speaker sets}

Speakers were divided into separate sets for training, calibration, evaluation and adaptation, shown in Table 1.

Table 1: The size of the sets used in the experiments.

\begin{tabular}{|c|c|}
\hline Speaker set & No. speakers \\
\hline Training child & 60 \\
Evaluation child & 60 \\
Development child & 48 \\
Adaptation child & 30 \\
Training adult & 60 \\
Evaluation adult & 60 \\
\hline
\end{tabular}

\section{Normalization and Adaptation}

A recognizer trained on adult speech is likely to have lower performance for children than for adults. One contributing factor is difference in vocal tract length. This may be compensated for during feature extraction by vocal tract length normalization (VTLN) which rescales the frequency axis of the spectral analysis [8].

VTLN is normally performed on speech sampled at the same frequency as the training speech. A piece-wise linear scaling is often used to map the high and low frequency limits onto themselves, see left part of Figure 1. However, this method does not use information in the spectral region above 
the recognizer's cut-off frequency. An alternative would be to use linear scaling to include spectral parts from above into the recognizer's analysis region; see right part of Figure 1. This requires extended bandwidth in the test speech compared to the analysis bandwidth of the recognizer.
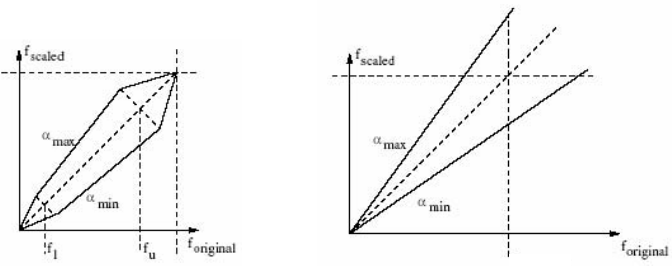

Figure 1: Piece-wise linear- (left) and linear (right) frequency warping algorithms.

Model adaptation to new speakers or environment conditions is commonly performed by the maximum a posteriori (MAP) [9] and the maximum likelihood linear regression (MLLR) [10] algorithms. In MAP adaptation, weighting is done between the old parameter set of the model and a maximum likelihood estimate based on the adaptation set. A key feature of MAP is that it only adapts models that are represented in the adaptation data. MLLR adaptation estimates a linear transform of model parameters to optimize the likelihood of the adaptation data. The method permits classes of models to share common transforms. Using classes enables adapting models that were not seen in the adaptation data. However, care must be taken to avoid clustering models that should not be adapted in a similar manner.

\section{Experiments}

Experiments were conducted on a connected digit task. Each digit was modeled as an HMM with the number of states set to three times the number of phonemes in the word. Observation probability density functions were modeled using Gaussian mixture models (GMM). The number of mixtures of each state was 8 based on a development set of speakers.

Feature extraction from the speech signal was performed each $10 \mathrm{~ms}$ on $25 \mathrm{~ms}$ Hamming windows. As features 13 MFCCs including energy, and their first and second order time derivatives were used. The MFCCs were computed using the cosine transform on a 26-channel mel filter bank in the range corresponding to the interval 0 to $8 \mathrm{kHz}$.

Models were trained on children, adults and the union of the two training sets. The training procedure is a modification of the RefRec script, developed in COST 249 [11]. Models are initialized in three successive training steps: flat start, time aligned data and cloning of context free models. All training and recognition experiments were implemented in the HTK speech recognition software package [12].

A correlation analysis was performed to rank the impact of some features on the word error rate of the recognizer. The features investigated were age, height, gender, fundamental frequency (F0), and the first and second formants. F0 was determined as the average of all frames during voiced speech, excluding creaky and whispery voice. Formant tracks were sampled at the mid point of vowels and averaged over all vowels for each child. These tracks were automatically tracked and manually corrected as necessary. Gender was represented by values -1 for girls and 1 for boys.
Age-dependent adaptation was performed using the adaptation set of 30 speakers. This set was divided into five categories, one for each age in the interval from 4 to 8 . Each category consisted of six children.

Height adaptation was done by grouping the adaptation set into five height categories of roughly equal number of speakers.

Adaptation of an adult model towards children in general was also performed. In this case 60 utterances were randomly chosen from the adaptation set to match the size of the age dependent adaptation data.

Vocal tract length normalization was performed in two ways; on groups of children and individually for each utterance. Individual scaling factors per utterance were estimated by optimizing the acoustic score against the adult models. In the case of a general scaling factor for children, direct optimization of the word error rate for the adaptation set of speakers was used. Linear VTLN was also attempted in order to take advantage of the higher bandwidth of the children recordings.

A comparison between word models and phoneme models was also carried out. In this experiment three-state triphones with 32 mixtures was used. State tying was performed based on a decision tree with questions regarding phonetic context in the same manner as in RefRec [11].

\section{Results}

Figure 2 shows the performance on adult and children's speech for each of the three acoustic models. As expected, performance was lower for mismatched than for matched conditions. In the matched cases, adult speech performed better than children's speech. The performance of the combined model was very close to those of the two matched conditions.

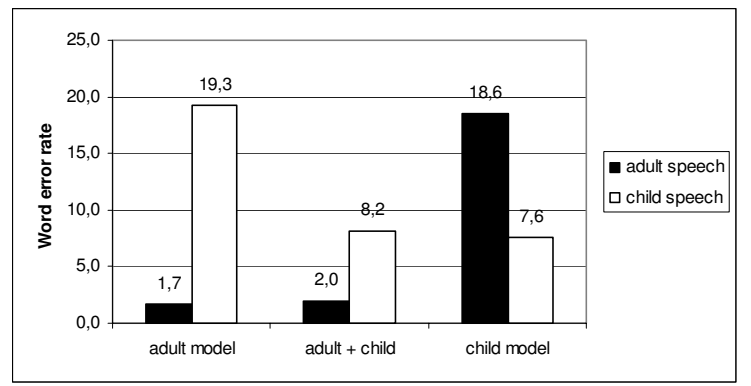

Figure 2: The performance of a digit recognizer with acoustic models trained on adults, children and the union of the two sets of speakers.

Table 2 shows the correlation coefficient between some features of the child and the error rate of the recognizer. Gender was only weakly correlated which suggests that no discrimination between boys and girls is needed for young children. Height showed a high correlation with the word error rate which is supportive evidence for applying VTLN in speech recognition. Age is correlated with many aspects of the child for instance height and the time available to learn to speak. Age was also found to have a high correlation with the word error rate. Thereby asking for the speakers' age might be a vital key to perform some kind of speaker adaptation. 
Table 2. Correlation between some speaker characteristic features and word error rate. The results were computed on a preliminary version of the database and are therefore not comparable to other results in the paper.

\begin{tabular}{|c|c|}
\hline Feature & Correlation \\
\hline Age & -0.51 \\
Height & -0.49 \\
Average F2 (Hz) & 0.40 \\
Average F0 (Hz) & 0.36 \\
Gender & -0.05 \\
Average F1 $(\mathrm{Hz})$ & 0.05 \\
\hline
\end{tabular}

Performance for each age group using the adult and the ageindependent child models is shown in Figure 3. The standard deviation is larger for the adult model and in both cases increases significantly with younger age. Older children are more correctly recognized for both model types. In the case of adult models a natural explanation is the further developed speech characteristics of older children towards adults. For the child models the lower performance of younger speakers is possibly due to their larger speech variability.

There is a clear decrease in error rate going from the 4 and 5 year old children to the older ages even when using the models trained on children. In this case the physical mismatch is smaller than for the adult models and therefore other sources of mismatch becomes more important. The results might suggest that articulation develops especially rapidly between the ages 5 and 6 years.

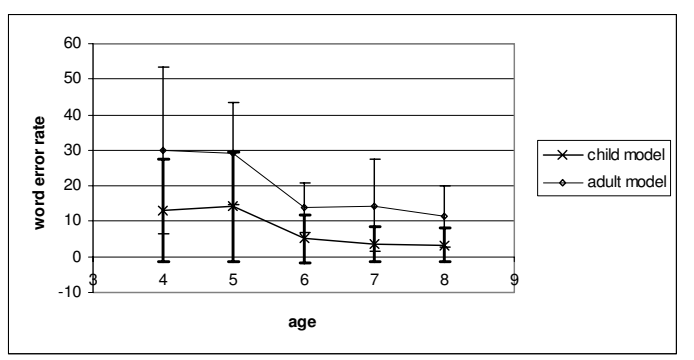

Figure 3: Word error rates using adult and ageindependent child models as a function of age.

The results of several adaptation and normalization techniques are shown in Table 3. Experiments on linear VTLN were not completed since the method turned out to be unexpectedly sensitive to the acoustic content of non-speech segments. All the other methods significantly decreased the original adult model error rate of $19.3 \%$. MAP and MLLR were more successful than piece-wise linear VTLN. It was also better to adapt to children in general than to age or height groups even though a roughly equal number of utterances was used in both cases. Training directly on the adaptation set resulted in a word error rate of $25.7 \%$.

The performance of the utterance-based VTLN method was slightly better than using a global warping factor for all children estimated on the adaptation set.
Table 3. The word error rate of normalization techniques applied to an adult model and models adapted towards children grouped by age, height and as a whole.

\begin{tabular}{|c|c|c|c|c|}
\cline { 2 - 4 } \multicolumn{1}{c|}{} & \multicolumn{3}{c|}{ Grouped adaptation data } & \multicolumn{1}{c}{} \\
\hline Method & Age & Height & Children & Utterance \\
\hline VTLN & 14.9 & 15.4 & 14.3 & 13.6 \\
MAP & 12.5 & 11.6 & 11.7 & \\
MLLR & 12.1 & 11.4 & 11,4 & \\
MLLRMAP & 11.3 & 11.5 & 9.5 & \\
\hline
\end{tabular}

MLLR was more successful than VTLN in decreasing the word error rate for all age groups as Figure 4 shows. One explanation might be that MLLR is able to compensate for other factors than the vocal tract length alone. Another explanation might be that VTLN introduces spectral distortion of the recording. Furthermore the match of the silence model is likely to degrade due to the contraction of the spectra.

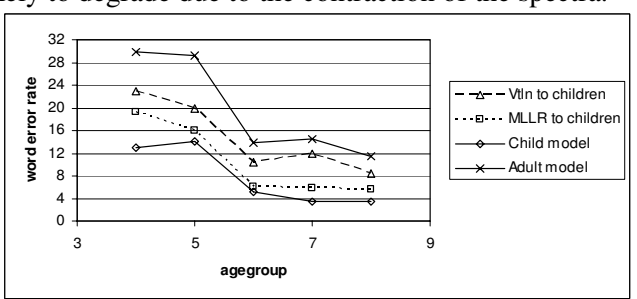

Figure 4: Detailed comparison between the word error rate as a function of age for piece-wise linear VTLN and MLLR to children as a group.

Linear and piece-wise linear VTLN show similar results at moderate warping factors, Figure 5. However, for warping factors below 0.7 linear VTLN performs better.
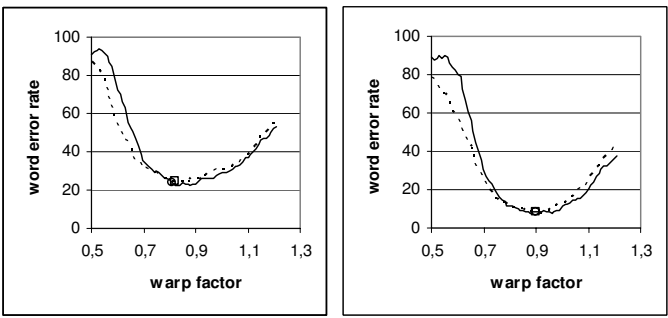

Figure 5: Word error rate as a function of warping factor for four and eight year old children (left and right respectively). Piece-wise linear VTLN is shown as a, solid line and linear VTLN as a dashed line. A marker shows the optimal warping factor based on the word error rate on the adaptation set.

No visible improvement was found for age-dependent models created by adapting the child model. Probably the amount of training data was sufficient for the task.

Recognition of digit strings using tied state triphone models gave a lower word error rate than using whole word models. This was true both for adults and children as is shown in Figure 6. 


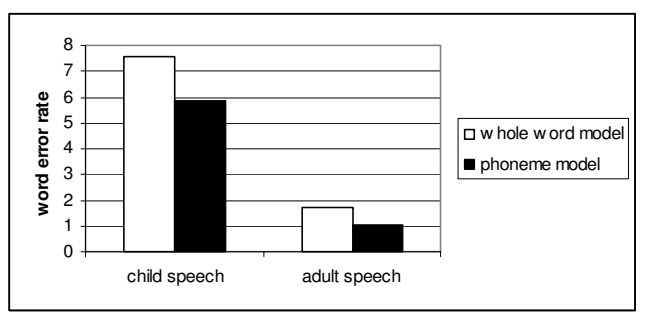

Figure 6: Word error rate of whole word models and tied triphone models. No mismatched cases are included.

\section{Discussion}

An undesirable effect of VTLN is that also non-speech signals are warped. It turned out that the optimal warp factor in several cases compensated for varying background noise instead of vocal tract length. An example is shown in Figure 7. The piece-wise linear algorithm was more robust to this effect due to its increased spectral distortion at warp factors deviating from 1.0. This problem would be avoided by performing warping on the models instead of the input signal, in which case different warping factors are applied to speech and non-speech segments, as well as for various phonemes.

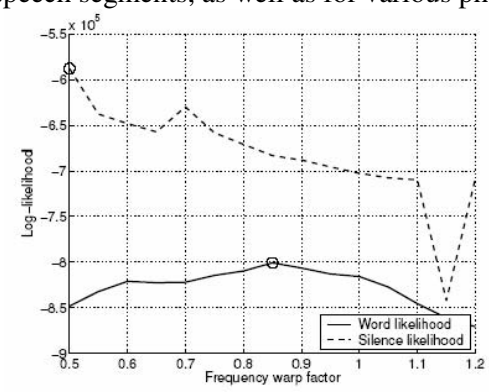

Figure 7: Likelihood scores as functions of linear VTLN warping factor for separate non-speech (dashed) and speech segments (solid) of an example utterance.

Modeling words by concatenating triphone models turned out to result in a higher performance than whole word models. This may be due to a more detailed alignment in the second initialization of the phoneme models than for the whole word models. Another possible cause is the state tying performed on phonetic context. As a consequence of using phonemes it also became possible to increase the number of mixtures from 16 to 32 terms per state. These results are somewhat preliminary and further experiments are therefore planned in this area.

\section{Conclusions}

Poor performance using adult models was improved significantly by adaptation and vocal tract length normalisation but not to the same level as training on children.

A combined model trained on adult and children's speech turned out to perform almost as well as dedicated models for each group of speakers.

The performance of the piece-wise linear VTLN algorithm is limited by its inherent penalty of the low warp factors necessary for children's speech. Both linear and piece- wise linear algorithms would probably perform better by adapting the models rather than normalization of the input signal.

Phoneme-based models gave better results than wordbased models, even for small vocabularies.

\section{Acknowledgements}

Data collection of children's speech and the experiments were made as a part of the EU project PF-STAR. Phonetic analysis was performed by Martina Huber, Lena Måhl adapted the RefRec recognizer, and Anders Schiöld implemented the VTLN scheme as a part of their thesis projects.

\section{References}

[1] Li, Q. and Russel, M. "An Analysis of the Causes of Increased Error Rates in Children's Speech Recognition", ICSLP 2002, Denver, pp. 2337-2340.

[2] Giuliani D. Gerosa M., Brugnara F., "Speaker Normalization through Constrained MLLR Based Transforms", ICSLP 2004.

[3] Giuliani D., Gerosa M., "Investigating Recognition of Children's Speech", ICASSP 2003, v2 pp. 137-140.

[4] Potamianos A., Narayanan S., "Robust Recognition of Children's Speech", IEEE Trans. Speech and Audio Proc., 11(6):603-616, 2003.

[5] Großkopf, B., Marasek, K., v. d. Heuvel, H., Diehl, F., Kiessling, A., "SpeeCon - speech data for consumer devices: Database specification and validation", Second International Conference on Language Resources and Evaluation 2002.

[6] Batliner A., Blomberg M., D'Arcy S., Elenius D., Giuliani D., Gerosa M., Hacker C., Russel M., Steidl S. Wong M.,"The PF_STAR Children's Speech Corpus", To appear in Interspeech 2005.

[7] Rudnicky, A. I., Polifroni, Thayer, E H., and Brennan, R. A. "Interactive problem solving with speech", J. Acoust. Soc. Amer., Vol. 84, 1988, p S213(A).

[8] Lee L. and Rose R. C., "Speaker normalization using efficient frequency warping procedures", in Proc. ICASSP, 1996, pp. 353-356.

[9] Gauvain J., "Maximum a posteriori estimation for multivariate Gaussian mixture observations of Markov chains," IEEE Transactions on Speech and Audio Processing, vol. 2, no. 2, pp. 291-298, April 1994.

[10] Leggetter C. and Woodland P., "Maximum likelihood linear regression for speaker adaptation of continuous density hidden Markov models," Computer Speech and Language, vol. 9, no. 2, pp. 171-185, April 1995.

[11] B. Lindberg, F.T. Johansen, N. Warakagoda, G. Lehtinen, Z. Kacic, A. Zgank, K. Elenius, G. Salvi, "A noise robust multilingual reference recogniser based on SpeechDat(II)", ICSLP 2000, v3, pp 370-373.

[12] Young S., Evermann G., Kershaw D., Gareth M., Odell J., Ollason D., Povey D., Valtchev V., Woodland P., "HTK book", Cambridge University Engineering Department 2002. 